\title{
On Global Attractors for a Class of Parabolic Problems
}

\author{
Rodiak Figueroa-López* and German Lozada-Cruz \\ ${ }^{1}$ Departamento de Matemática, IBILCE, UNESP - Universidade Estadual Paulista, 15054-000 São José do Rio Preto, São Paulo, Brasil
}

Received: 10 Mar. 2013, Revised: 11 Jul. 2013, Accepted: 13 Jul. 2013

Published online: 1 Mar. 2014

\begin{abstract}
This paper is devoted to study the existence of global attractor in $H_{0}^{1}(\Omega)$ and uniform bounds of it in $L^{\infty}(\Omega)$ for a class of parabolic problems with homogeneous boundary conditions wich involves a uniform strongly elliptic operator of second order in the domain $\Omega \subset \mathbb{R}^{n}$. The main tools used to prove the existence of global attractor are the techniques used in Hale [8] and Cholewa [5], and for the uniform bound of the attractor we use the Alikakos-Moser iteration procedure [1].
\end{abstract}

Keywords: Parabolic equation, sectorial operator, global attractor, uniform boundness

\section{Introduction}

Today, the concept of global attractor is a very useful tool for studying the asymptotic behavior of differential equations, that is, an attractor is a nonempty subset $\mathscr{A}$ of the phase space which is compact, invariant under the flow and attracts every bounded set under the semigroup associated to the PDE (for more details see [8], [10] and the reference therein). For example, suppose that the parabolic problem

$$
\left\{\begin{array}{l}
u_{t}=L u+f(u), t>0, x \in \Omega \\
u(t, x)=0, \quad t>0, x \in \partial \Omega \\
u(0, \cdot)=u^{0}(\cdot) \in H_{0}^{1}(\Omega),
\end{array}\right.
$$

models a certain phenomenon. Here $\Omega \subset \mathbb{R}^{n}$ is a bounded domain with smooth boundary $\partial \Omega, L$ is second order elliptic operator given by

$$
L u=\sum_{i, j=1}^{n} \frac{\partial}{\partial x_{i}}\left(a_{i j}(x) \frac{\partial u}{\partial x_{j}}\right)+\sum_{j=1}^{n} b_{j}(x) \frac{\partial u}{\partial x_{j}}+(c(x)+\lambda) u
$$

with coefficients $a_{i j}, b_{j}, c: \bar{\Omega} \rightarrow \mathbb{R}$ smooth, $a_{i j}=a_{j i}, i, j=$ $1, \cdots, n, \lambda \in \mathbb{R}$ and $f: \mathbb{R} \rightarrow \mathbb{R}$ is a nonlinear function of class $C^{2}(\mathbb{R})$ satisfying the condition

$$
\left|f^{\prime}(s)\right| \leqslant C\left(1+|s|^{\gamma-1}\right), \forall s \in \mathbb{R},
$$

where $1 \leqslant \gamma<\frac{n+2}{n-2}$ if $n \geqslant 3$, and $\gamma \geqslant 1$ if $n=2$, and

$$
\limsup _{|u| \rightarrow \infty} \frac{f(u)}{u} \leqslant 0 \text {. }
$$

We assume that $L$ is uniformly strongly elliptic operator, that is, there is a constant $\vartheta>0$, such that

$$
\sum_{i, j=1}^{n} a_{i j}(x) \xi_{i} \xi_{j} \geqslant \vartheta\left(\sum_{k=1}^{n} \xi_{k}^{2}\right)
$$

for all $x \in \Omega$ and $\xi=\left(\xi_{1}, \ldots, \xi_{n}\right) \in \mathbb{R}^{n}$.

The main goal of this work is to prove the existence and uniform boundedness of global attractor for the initialboundary value problem (1) where the operator $L$ presents lower order terms. The presence of such that terms in the operator $L$, in particular $b_{j} u_{x_{j}}$ makes it difficult to obatins directly the Lyapunov function as in the works of Hale [8].

Let $X=L^{2}(\Omega)$ be a Hilbert space and define the linear operator $A: D(A) \subset X \rightarrow X$ by

$$
\begin{aligned}
D(A) & =H^{2}(\Omega) \cap H_{0}^{1}(\Omega), \\
A u & =-L u, \quad \forall u \in D(A) .
\end{aligned}
$$

There are many studies on PDE's for (1) with the operator $A$ being self-adjoint (see [5], [8], [9]). In our case the operator $A$ is not self-adjoint.

We will show that the operator $A$ is sectorial and assuming that $\lambda$ is chosen such that $\operatorname{Re} \sigma(A)>0$, we can define the fractional powers $A^{\alpha}$ and the corresponding fractional power spaces $X^{\alpha}:=D\left(A^{\alpha}\right), \alpha>0$, endowed with the graph norm (see [5, Section 1.3.3]). $X^{\alpha}$ is a Hilbert space with the inner product $\langle\varphi, \psi\rangle_{\alpha}=\int_{\Omega}\left(A^{\alpha} \varphi\right)\left(A^{\alpha} \psi\right)$. Then, $\quad X^{1}=D(A)$, $X^{0}=L^{2}(\Omega)$ and $X^{1 / 2}=H_{0}^{1}(\Omega)$.

\footnotetext{
* Corresponding author e-mail: rodiak@ibilce.unesp.br
} 
With this notation, the problem (1) can be written in the abstract form

$$
\left\{\begin{array}{l}
\dot{u}+A u=F(u) \\
u(0)=u^{0} \in X^{1 / 2}
\end{array}\right.
$$

where $F: X^{1 / 2} \rightarrow X$ is the Nemytskii operator given by $F(u(t)) x=f(u(x, t))$. From Henry's theory [9], the equation (3) defines a semigroup $T(t, \cdot)$ on $X^{1 / 2}$, for $t \geqslant 0$.

Next, we describe the contents of the paper. In section 2 we will show that the operator $A$ is sectorial and that $F$ and $F^{\prime}$ is is locally Lipschitz continuous. In section 3 we prove the existence of local and global solution of (1) and finally in section 4 we prove the existence of the global attractor and the uniform boundedness for it.

\section{The sectoriality of operator $A$}

Firstly, we will be prove the following.

Lemma 1.Let $A_{0}: D\left(A_{0}\right)=D(A) \subset X \rightarrow X$ be a linear operator given by

$$
A_{0} u=-\sum_{i, j=1}^{n} \frac{\partial}{\partial x_{i}}\left(a_{i j}(x) \frac{\partial u}{\partial x_{j}}\right) .
$$

Then, $A_{0}$ is sectorial in $X$.

Furthermore, there is a constant $\rho>0$ such that

$$
\|u\|_{H^{2}(\Omega)} \leqslant \rho\left\|A_{0} u\right\|_{X}, \forall u \in X
$$

Proof. Let $\lambda_{0}>0$. We will show that $A_{0}+\lambda_{0} I$ é setorial, then by the Remark 1.3.1 in [5, p.32] it follows that $A_{0}$ is sectorial. Indeed, we have $D\left(A_{0}\right)=D\left(A_{0}+\lambda_{0} I\right)$ and for $u, v \in D\left(A_{0}\right)$,

$$
\begin{aligned}
\left\langle\left( A_{0}\right.\right. & \left.\left.+\lambda_{0} I\right) u, v\right\rangle_{X}=\sum_{i, j=1}^{n} \int_{\Omega} a_{j i}(x) \frac{\partial v}{\partial x_{i}} \frac{\partial u}{\partial x_{j}} d x+\lambda_{0} \int_{\Omega} u v d x \\
& =-\sum_{i, j=1}^{n} \int_{\Omega} u \frac{\partial}{\partial x_{j}}\left(a_{j i}(x) \frac{\partial v}{\partial x_{i}}\right) d x+\lambda_{0} \int_{\Omega} u v d x \\
& =\left\langle u,\left(A_{0}+\lambda_{0} I\right) v\right\rangle_{X}
\end{aligned}
$$

thus, $A_{0}+\lambda_{0} I$ is symmetric operator.

Using the Sobolev embeddings (see [5, p. 23]) and the density of $C_{0}^{\infty}(\Omega)$ in $L^{p}(\Omega), 1 \leqslant p<\infty$, we conclude that $A_{0}+\lambda_{0} I$ is densely defined in $X$. Therefore, $D\left(A_{0}\right)$ is densely defined in $X$.

Now, let $f \in X$ be such that $\left(A_{0}+\lambda_{0} I\right) u=f$. Following the Example 3 in [4, p. 294] taking $a_{0}=\lambda_{0}>0$, we conclude that there is $u \in D\left(A_{0}\right)$ satisfying $\left(A_{0}+\lambda_{0} I\right) u=f$. Thus, $R\left(A_{0}+\lambda_{0} I\right)=X$. Using Theorem 13.11 item $(d)$ in $[11$, p.334] we conclude that $A_{0}+\lambda_{0} I$ is selfadjoint.
Also, for $u \in D\left(A_{0}\right)$, we obtain

$$
\begin{gathered}
\left\langle\left(A_{0}+\lambda_{0} I\right) u, u\right\rangle_{X}=\sum_{i, j=1}^{n} \int_{\Omega} a_{i j}(x) \frac{\partial u}{\partial x_{j}} \frac{\partial u}{\partial x_{i}} d x+\lambda_{0} \int_{\Omega} u^{2} d x \\
\geqslant \vartheta \sum_{k=1}^{n} \int_{\Omega}\left|\frac{\partial u}{\partial x_{k}}\right|^{2} d x+\lambda_{0} \int_{\Omega}|u|^{2} d x \geqslant \lambda_{0}\|u\|_{X}^{2} .
\end{gathered}
$$

Here we have used (2). Now, by density it follows that $A_{0}+\lambda_{0} I$ is bounded below and by Proposition 1.3.3 in [5, p. 39] $A_{0}+\lambda_{0} I$ is sectorial in $X$.

Finally using the results of elliptic regularity (taking $m=1, k=0, p=2$ and $B_{j}=I, j=1$ in [12, p. 14]), we have

$$
\|u\|_{H^{2}(\Omega)} \leqslant \rho\left\|A_{0} u\right\|_{L^{2}(\Omega)},
$$

where $\rho>0$ and $\operatorname{ker}\left(A_{0}\right)=\{0\}$. This last result is extend to $X$ by density of $D\left(A_{0}\right)$.

Lemma 2.The operator $A$ is sectorial in $X$.

Proof. Let $B: D(B) \subset X \rightarrow X$ be a linear operator given by

$$
-B u=\sum_{j=1}^{n} b_{j}(x) \frac{\partial u}{\partial x_{j}}+(c(x)+\lambda) u,
$$

where $D(B)=\left\{u \in L^{2}(\Omega): B u \in L^{2}(\Omega), u=0\right.$ in $\left.\partial \Omega\right\}=$ $H_{0}^{1}(\Omega)$.

By Gagliardo-Nirenberg's inequality (Theorem 10.1 in [7, p. 27]) with $m=r=p=2, j=1$ and $\theta=1 / 2$, we have

$$
\|u\|_{H_{0}^{1}(\Omega)} \leqslant C\|u\|_{H^{2}(\Omega)}^{\frac{1}{2}}\|u\|_{L^{2}(\Omega)}^{\frac{1}{2}},
$$

where $C$ is a constant.

By other side, from the Young's inequality and (5), we obtain

$$
\|u\|_{H_{0}^{1}(\Omega)} \leqslant \frac{\varepsilon}{2}\|u\|_{H^{2}(\Omega)}+\frac{C^{2}}{2 \varepsilon}\|u\|_{L^{2}(\Omega)}, \forall \varepsilon>0 .
$$

Substituting (4) in (6) we get

$$
\|u\|_{H_{0}^{1}(\Omega)} \leqslant \frac{\rho \varepsilon}{2}\left\|A_{0} u\right\|_{L^{2}(\Omega)}+\frac{C^{2}}{2 \varepsilon}\|u\|_{L^{2}(\Omega)} .
$$

Thus,

$$
\|B u\|_{X} \leqslant \frac{\tau \rho \varepsilon}{2}\left\|A_{0} u\right\|_{L^{2}(\Omega)}+\frac{\tau C^{2}}{2 \varepsilon}\|u\|_{L^{2}(\Omega)},
$$

where in the last inequality we have used (7) and $\tau=\max \left\{\max _{x \in \bar{\Omega}}\left|b_{j}(x)\right|, \max _{x \in \bar{\Omega}}|c(x)+\lambda|\right\}$.

Observe that $A=A_{0}+B$ where $A_{0}$ is sectorial in $X$, $D\left(A_{0}\right) \subset D(B)$. Therefore, by Theorem 2.6.3 in [12, p. 69] and (8) follows that $A$ is a sectorial in $X$. $\square$

Now, we prove that $F$ is locally Lipschitz continous.

Lemma 3.If $f: \mathbb{R} \rightarrow \mathbb{R}$ is a function of class $C^{1}(\mathbb{R})$, the assumption (C) is satisfied and $n \geqslant 1$ then $F: X^{1 / 2} \rightarrow X$ is locally Lipschitz continous. 
Proof. The case $n=1$ was proved in [8, p.75]. Now, let $n \geqslant 2$. Using the mean value theorem and assumption (C), we have

$$
\begin{aligned}
|f(s)-f(r)| & =\left|f^{\prime}(\theta s+(1-\theta) r)\right||s-r|, 0 \leqslant \theta \leqslant 1 \\
& \leqslant C\left(1+|\theta s+(1-\theta) r|^{\gamma-1}\right)|s-r| \\
& \leqslant C_{1}\left(1+|s|^{\gamma-1}+|r|^{\gamma-1}\right)|s-r|
\end{aligned}
$$

where $C_{1}=C_{1}(\gamma), 1 \leqslant \gamma<\frac{n+2}{n-2}$ if $n \geqslant 3$ and $\gamma \geqslant 1$ if $n=2$.

Now, we will to show that for $p>1$ satisfying $p \gamma=\frac{2 n}{n-2}$, the function $F: L^{\frac{2 n}{n-2}}(\Omega) \rightarrow L^{p}(\Omega)$ is locally Lipschitz continuous. Indeed, using (9) we get

$$
\begin{aligned}
\| F(u) & -F(v) \|_{L^{p}(\Omega)}^{p}=\int_{\Omega}|f(u(x))-f(v(x))|^{p} d x \\
\leqslant & C_{1} \int_{\Omega}\left(1+|u(x)|^{\gamma-1}+|v(x)|^{\gamma-1}\right)^{p}|u(x)-v(x)|^{p} d x \\
\leqslant & C_{2}\left(\int_{\Omega}|u(x)-v(x)|^{p \gamma} d x\right)^{\frac{1}{\gamma}} \\
& \times\left(\int_{\Omega}\left(1+|u(x)|^{p(\gamma-1)}+|v(x)|^{p(\gamma-1)}\right)^{r} d x\right)^{\frac{1}{r}} \\
\leqslant & C_{3}\left(\int_{\Omega}|u(x)-v(x)|^{\frac{2 n}{n-2}} d x\right)^{\frac{p(n-2)}{2 n}} \\
& \times\left(\int_{\Omega}\left(1+|u(x)|^{p(\gamma-1) r}+|v(x)|^{p(\gamma-1) r}\right) d x\right)^{\frac{1}{r}},
\end{aligned}
$$

where in the second inequality we have used the Hölder inequality for $\gamma$ and $r$, with $\frac{1}{r}=1-\frac{p(n-2)}{2 n}$ because $u, v \in L^{\frac{2 n}{n-2}}(\Omega)=L^{p \gamma}(\Omega)$. Since $\gamma$ and $r$ are conjugate we have $p(\gamma-1) r=p \gamma$, thus $(u-v)^{p} \in L^{\gamma}(\Omega)$, and since $u^{p(\gamma-1)}, \quad v^{p(\gamma-1)} \in L^{r}(\Omega) \quad$ we have also $1+u^{p(\gamma-1)}+v^{p(\gamma-1)} \in L^{r}(\Omega)$. Then,

$$
\begin{aligned}
& \|F(u)-F(v)\|_{L^{p}(\Omega)}^{p} \leqslant C_{4}\|u-v\|_{L^{p \gamma}(\Omega)}^{p} \\
& \quad \times\left(1+\|u\|_{L^{p \gamma(\Omega)}}^{p \gamma}+\|v\|_{L^{p \gamma}(\Omega)}^{p \gamma}\right)^{\frac{1}{r}} \\
& \leqslant C_{5}\|u-v\|_{L^{\frac{2 n}{n-2}(\Omega)}}^{p}\left(1+\|u\|_{L^{\frac{2 n}{n-2}(\Omega)}}+\|v\|_{L^{\frac{2 n}{n-2}(\Omega)}}\right)^{p(\gamma-1)} .
\end{aligned}
$$

Therefore,

$$
\begin{aligned}
\|F(u)-F(v)\|_{L^{p}(\Omega)} & \leqslant C_{6}\|u-v\|_{L^{\frac{2 n}{n-2}}(\Omega)} \\
& \times\left(1+\|u\|_{L^{\frac{2 n}{n-2}(\Omega)}}+\|v\|_{L^{\frac{2 n}{n-2}(\Omega)}}\right)^{\gamma-1} .
\end{aligned}
$$

In particular, for $p=2$, using the immersions $H_{0}^{1}(\Omega) \hookrightarrow$ $L^{\sigma}(\Omega), \sigma \in\left[1, \frac{2 n}{n-2}\right]$ for $n \geqslant 3$ and $H_{0}^{1}(\Omega) \hookrightarrow L^{\sigma}(\Omega), \sigma \in$ $[1, \infty)$ for $n=2$, we have

$$
\|F(u)-F(v)\|_{X} \leqslant C_{7}\|u-v\|_{X^{1 / 2}}\left(1+\|u\|_{X^{1 / 2}}+\|v\|_{X^{1 / 2}}\right)^{\gamma-1} .
$$

Finally, given $\delta>0$ such that $u, v \in X^{1 / 2}$ with $\|u\|_{X^{1 / 2}},\|v\|_{X^{1 / 2}} \leqslant \delta$ we have

$$
\|F(u)-F(v)\|_{X} \leqslant C_{\delta, \gamma}\|u-v\|_{X^{1 / 2}},
$$

where $C_{\delta, \gamma}=C_{7}(1+2 \delta)^{\gamma-1}$. Therefore $F$ is locally Lipschitz continuous.

Lemma 4.Let $f: \mathbb{R} \rightarrow \mathbb{R}$ be a function of class $C^{2}(\mathbb{R})$ satisfying (C). Then, $F: X^{1 / 2} \rightarrow X$ is a function of class $C^{1} \quad$ with $F^{\prime}(u) \in \mathscr{L}\left(X^{1 / 2}, X\right)$ given by $\left[F^{\prime}(u) h\right](x)=f^{\prime}(u(x)) h(x)$, for all $u, h \in X^{1 / 2}$.

Moreover, there is a constante $C>0$ such that

$$
\begin{aligned}
\| F^{\prime}(u)-F^{\prime}(v) & \left\|_{\mathscr{L}\left(X^{1 / 2}, X\right)} \leqslant C\right\| u-v \|_{X^{1 / 2}} \\
& \times\left(1+\|u\|_{X^{1 / 2}}+\|v\|_{X^{1 / 2}}\right)^{\gamma-2},
\end{aligned}
$$

for all $u, v \in X^{1 / 2}$.

Proof. First we obtain the Gateaux differential of the Nemytskii's operator. Thus, for $u, h \in X^{1 / 2}$ we have

$$
\begin{aligned}
\delta F(u, h)(x) & =\lim _{t \rightarrow 0} \frac{f(u(x)+\operatorname{th}(x))-f(u(x))}{t} \\
& =\lim _{t \rightarrow 0} \int_{0}^{1} \frac{f^{\prime}(u(x)+\operatorname{sth}(x)) \operatorname{th}(x)}{t} d s \\
& =\int_{0}^{1} f^{\prime}(u(x)) h(x) d s=f^{\prime}(u(x)) h(x),
\end{aligned}
$$

where we have used the Dominated Convergence Theorem of Lebesgue and the fact that $f \in C^{2}(\mathbb{R})$ with $s=s(x) \in$ $[0,1]$.

From assumption $(\mathrm{C})$, we obtain

$$
\left|f^{\prime \prime}(s)\right| \leqslant C_{\gamma}\left(1+|s|^{\gamma-2}\right),
$$

where $s \in \mathbb{R}$ with $2 \leqslant \gamma \leqslant \frac{n+2}{n-2}$ if $n \geqslant 3$ and $\gamma \geqslant 2$ if $n=2$.

Similarly, as was done for (9) using (CC), we have

$$
\left|f^{\prime}(s)-f^{\prime}(r)\right| \leqslant C_{1, \gamma}\left(1+|s|^{\gamma-2}+|r|^{\gamma-2}\right)|s-r|,
$$

where $2 \leqslant \gamma<\frac{n+2}{n-2}$ if $n \geqslant 3$ and $\gamma \geqslant 2$ if $n=2$.

Now, we can show that $F$ is of class $C^{1}$ in the sense of Fréchet. We begin by defining

$$
\Phi(x):=F(u+h)(x)-F(u)(x)-f^{\prime}(u(x)) h(x),
$$

for $u, h \in L^{\frac{2 n}{n-2}}$ and $x \in \Omega$. Thus, for $p>1$ such that $p \gamma=$ $\frac{2 n}{n-2}$ and (11) we obtain

$$
\begin{aligned}
& \|\Phi\|_{L^{p}(\Omega)}^{p}=\int_{\Omega}\left|\int_{0}^{1}\left[f^{\prime}(u(x)+\operatorname{sh}(x))-f^{\prime}(u(x))\right] d s h(x)\right|^{p} d x \\
& \leqslant C_{1, \gamma}^{p} \int_{\Omega}\left|\int_{0}^{1}\left(1+|u(x)+\operatorname{sh}(x)|^{\gamma-2}+|u(x)|^{\gamma-2}\right)\right| \operatorname{sh}(x) \mid \\
& \quad \times\left.|h(x)| d s\right|^{p} d x \\
& \leqslant C_{2, \gamma}^{p} \int_{\Omega}|h(x)|^{2 p}\left(1+|u(x)|^{\gamma-2}+|h(x)|^{\gamma-2}\right)^{p} d x \\
& \leqslant C_{3, \gamma}^{p} \int_{\Omega}|h(x)|^{2 p}\left(1+|u(x)|^{p(\gamma-2)}+|h(x)|^{p(\gamma-2)}\right) d x \\
& \leqslant C_{4, \gamma}^{p}\|h\|_{L^{p \gamma}(\Omega)}^{2 p}\left(\int_{\Omega}\left(1+|u(x)|^{p \gamma}+|h(x)|^{p \gamma}\right) d x\right)^{\frac{\gamma-2}{\gamma}} \\
& \leqslant C_{5, \gamma}^{p}\|h\|_{L^{p \gamma}(\Omega)}^{2 p}\left(1+\|u\|_{L^{p \gamma}(\Omega)}^{p \gamma}+\|h\|_{L^{p \gamma}(\Omega)}^{p \gamma}\right)^{\frac{\gamma-2}{\gamma}} \\
& \leqslant C_{6, \gamma}^{p}\|h\|_{L^{p \gamma}(\Omega)}^{2 p}\left(1+\|u\|_{L^{p \gamma}(\Omega)}+\|h\|_{L^{p \gamma}(\Omega)}\right)^{p(\gamma-2)},
\end{aligned}
$$


where we have used Hölder's inequality for $\frac{\gamma}{2}$ and $\frac{\gamma}{\gamma-2}$. Then,

$\|\Phi\|_{L^{p}(\Omega)} \leqslant C_{6, \gamma}\|h\|_{L^{p \gamma}(\Omega)}^{2}\left(1+\|u\|_{L^{p \gamma}(\Omega)}+\|h\|_{L^{p \gamma}(\Omega)}\right)^{\gamma-2}$.

Now, by the Sobolev embeddings we obtain

$\frac{\|\Phi\|_{L^{p}(\Omega)}}{\|h\|_{H_{0}^{1}(\Omega)}} \leqslant C_{7, \gamma}\|h\|_{H_{0}^{1}(\Omega)}\left(1+\|u\|_{H_{0}^{1}(\Omega)}+\|h\|_{H_{0}^{1}(\Omega)}\right)^{\gamma-2}$.

Therefore, $\frac{\|\Phi\|_{L^{p}(\Omega)}}{\|h\|_{H_{0}^{1}(\Omega)}} \rightarrow 0$ when $\|h\|_{H_{0}^{1}(\Omega)} \rightarrow 0$.

In particular, for $p=2$ the function $F$ is of class $C^{1}$ in the sense of Fréchet with $\left[F^{\prime}(u) h\right](x)=f^{\prime}(u(x)) h(x)$, for all $u, h \in H_{0}^{1}(\Omega)$.

Now we will show the estimate (10). In fact, for $u, v, h \in L^{p \gamma}(\Omega)$ denoting

$$
\Delta F(u, v):=F^{\prime}(u)-F^{\prime}(v)
$$

and using (11) we obtain

$$
\begin{aligned}
\| & \Delta F(u, v) h \|_{L^{p}(\Omega)}^{p}=\int_{\Omega}\left|f^{\prime}(u(x))-f^{\prime}(v(x))\right|^{p}|h(x)|^{p} d x \\
\leqslant & C_{1, \gamma}^{p} \int_{\Omega}\left(1+|u(x)|^{\gamma-2}+|v(x)|^{\gamma-2}\right)^{p}|u(x)-v(x)|^{p}|h(x)|^{p} d x \\
\leqslant & C_{8, \gamma}^{p} \int_{\Omega}\left(1+|u(x)|^{p(\gamma-2)}+|v(x)|^{p(\gamma-2)}\right)|u(x)-v(x)|^{p} \\
& \times|h(x)|^{p} d x\left(\int_{\Omega}\left(1+|u(x)|^{p(\gamma-2)}+|v(x)|^{p(\gamma-2)}\right)^{\theta} d x\right)^{\frac{1}{\theta}} \\
\leqslant & C_{9, \gamma}^{p}\|u-v\|_{L^{p \gamma}(\Omega)}^{p}\|h\|_{L^{p \gamma}(\Omega)}^{p} \\
& \times\left(1+\|u\|_{L^{p \theta(\gamma-2)}(\Omega)}^{p \theta(\gamma-2)}+\|v\|_{L^{p \theta(\gamma-2)}(\Omega)}^{p \theta(\gamma-2)}\right)^{\frac{1}{\theta}} \\
\leqslant & C_{10, \gamma}^{p}\|u-v\|_{L^{p \gamma}(\Omega)}^{p}\|h\|_{L^{p \gamma}(\Omega)}^{p} \\
& \times\left(1+\|u\|_{L^{p \theta(\gamma-2)}(\Omega)}+\|v\|_{L^{p \theta(\gamma-2)}(\Omega)}\right)^{p(\gamma-2)} \\
\leqslant & C_{10, \gamma}^{p}\|u-v\|_{L^{\frac{2 n}{n-2}}(\Omega)}^{p}\|h\|_{L^{\frac{2 n}{n-2}}(\Omega)}^{p} \\
& \times\left(1+\|u\|_{L^{\frac{2 n}{n-2}}(\Omega)}+\|v\|_{L^{\frac{2 n}{n-2}}(\Omega)}\right)^{p(\gamma-2)},
\end{aligned}
$$

where $\theta=\theta(\gamma)$ and we have used the Hölder's inequality for $\gamma, \gamma$ and $\theta$ with $\theta=\frac{\gamma}{\gamma-2}$, then $p(\gamma-2) \theta=p \gamma$ and $u^{p(\gamma-2)}, v^{p(\gamma-2)} \in L^{\theta}(\Omega)$.

Therefore,

$$
\begin{gathered}
\left\|F^{\prime}(u) h-F^{\prime}(u) h\right\|_{L^{p}(\Omega)} \leqslant C_{10, \gamma}\|u-v\|_{L^{\frac{2 n}{n-2}}(\Omega)}\|h\|_{L^{\frac{2 n}{n-2}}(\Omega)} \\
\times\left(1+\|u\|_{L^{\frac{2 n}{n-2}}(\Omega)}+\|v\|_{L^{\frac{2 n}{n-2}}(\Omega)}\right)^{\gamma-2} .
\end{gathered}
$$

Finally by the Sobolev embedding we obtain

$$
\begin{aligned}
\left\|F^{\prime}(u) h-F^{\prime}(u) h\right\|_{L^{p}(\Omega)} \leqslant C_{11, \gamma}\|u-v\|_{H_{0}^{1}(\Omega)} \\
\quad \times\left(1+\|u\|_{H_{0}^{1}(\Omega)}+\|v\|_{H_{0}^{1}(\Omega)}\right)^{\gamma-2}\|h\|_{H_{0}^{1}(\Omega)} .
\end{aligned}
$$

From this last inequality taking $p=2$ immediately follows (10). $\square$

Now let's get uniform bounds for the solutions of (3).
Lemma 5.Consider the problem (1) under all the hypothesis to get the existence of smooth solutions and satisfying

$$
u f(u) \leqslant \zeta u^{2}+\tau
$$

where $\zeta$ and $\tau$ are positive constants. Then,

$$
\sup _{t \geqslant 0}\left\|u\left(t, u^{0}\right)\right\|_{L^{\infty}(\Omega)} \leqslant \delta^{\frac{3}{2}} 2^{\frac{3 n}{4}+1} \max \left\{\left\|u^{0}\right\|_{L^{2}(\Omega)}, 1\right\}
$$

where $\delta>0$ is defined in (28) below.

Proof. We use Alikakos-Moser iteration (see [1] e [5]) which allows us to obtain estimates on $L^{\infty}(\Omega)$ for the solutions of parabolic equations of second order. Indeed, multiplying (1) by $u^{2^{k}-1}, k=1,2, \ldots$ and integrating over $\Omega$ we get

$$
\begin{aligned}
& \int_{\Omega} u_{t} u^{2^{k}-1} d x=\underbrace{\int_{\Omega} \sum_{i, j=1}^{n} \frac{\partial}{\partial x_{j}}\left(a_{i j}(x) \frac{\partial u}{\partial x_{i}}\right) u^{2^{k}-1} d x}_{=: \mathrm{I}} \\
& +\underbrace{\int_{\Omega} \sum_{i=1}^{n} b_{i}(x) \frac{\partial u}{\partial x_{i}} u^{2^{k}-1} d x}_{=: \mathrm{II}}+\int_{\Omega}(c(x)+\lambda) u^{2^{k}} d x \\
& +\int_{\Omega} f(u) u^{2^{k}-1} d x .
\end{aligned}
$$

For I, we have

$$
\begin{aligned}
\mathrm{I} & =-\int_{\Omega} \sum_{i, j=1}^{n} a_{i j}(x) \frac{\partial u}{\partial x_{i}} \frac{\partial\left(u^{2^{k}-1}\right)}{\partial x_{j}} d x \\
& =-\frac{\left(2^{k}-1\right)}{2^{2 k-2}} \int_{\Omega} \sum_{i, j=1}^{n} a_{i j}(x) \frac{\partial\left(u^{2^{k-1}}\right)}{\partial x_{i}} \frac{\partial\left(u^{2^{k-1}}\right)}{\partial x_{j}} d x .
\end{aligned}
$$

Similarly, for II, we have

$$
\begin{aligned}
\mathrm{II} & =\int_{\Omega} \sum_{i=1}^{n} b_{i}(x) u^{2^{k-1}-1} \frac{\partial u}{\partial x_{i}} u^{2^{k-1}} d x \\
& =\frac{1}{2^{k-1}} \int_{\Omega} \sum_{i=1}^{n} b_{i}(x) u^{2^{k-1}} \frac{\partial\left(u^{2^{k-1}}\right)}{\partial x_{i}} d x .
\end{aligned}
$$

Replacing (15) and (16) in (14) we get

$$
\begin{aligned}
& \frac{1}{2^{k}} \frac{d}{d t} \int_{\Omega} u^{2^{k}} d x=\frac{\left(1-2^{k}\right)}{2^{k-2}} \int_{\Omega} \sum_{i, j=1}^{n} a_{i j}(x) \frac{\partial\left(u^{2^{k-1}}\right)}{\partial x_{i}} \frac{\partial\left(u^{2^{k-1}}\right)}{\partial x_{j}} d x \\
& +2^{k} \int_{\Omega} u f(u) u^{2^{k}-2} d x+2 \int_{\Omega} \sum_{i=1}^{n} b_{i}(x) \frac{\partial\left(u^{2^{k-1}}\right)}{\partial x_{i}} u^{2^{k-1}} d x \\
& +2^{k} \int_{\Omega}(c(x)+\lambda) u^{2^{k}} d x .
\end{aligned}
$$


Now, using (2) and (12) we get

$$
\begin{aligned}
& \frac{d}{d t} \int_{\Omega} u^{2^{k}} d x \leqslant-\frac{\left(2^{k}-1\right)}{2^{k-2}} \vartheta \int_{\Omega} \sum_{i=1}^{n}\left[\frac{\partial\left(u^{2^{k-1}}\right)}{\partial x_{i}}\right]^{2} d x \\
& +2^{k} \int_{\Omega}\left(\zeta u^{2}+\tau\right) u^{2^{k}-2} d x+2 B_{\infty}^{*} \int_{\Omega} \sum_{i=1}^{n} \frac{\partial\left(u^{2^{k-1}}\right)}{\partial x_{i}} u^{2^{k-1}} d x \\
& +2^{k}\left(C_{\infty}^{*}(x)+\lambda\right) \int_{\Omega} u^{2^{k}} d x
\end{aligned}
$$

where $B_{\infty}^{*}:=\max _{1 \leqslant i \leqslant n} \sup _{x \in \Omega}\left|b_{i}(x)\right|$ and $C_{\infty}^{*}:=\sup _{x \in \Omega}|c(x)|$. Using Hölder's inequality in (17), we obtain

$$
\begin{aligned}
& \frac{d}{d t} \int_{\Omega} u^{2^{k}} d x \leqslant-\frac{\left(2^{k}-1\right)}{2^{k-2}} \vartheta \int_{\Omega} \sum_{i=1}^{n}\left[\frac{\partial\left(u^{2^{k-1}}\right)}{\partial x_{i}}\right]^{2} d x \\
& +2^{k} \zeta \int_{\Omega} u^{2^{k}} d x+2^{k} \tau \int_{\Omega} u^{2^{k}-2} d x \\
& +2 B_{\infty}^{*}\left(\int_{\Omega}\left[\sum_{i=1}^{n} \frac{\partial\left(u^{2^{k-1}}\right)}{\partial x_{i}}\right]^{2} d x\right)^{\frac{1}{2}}\left(\int_{\Omega}\left(u^{2^{k-1}}\right)^{2} d x\right)^{\frac{1}{2}} \\
& +2^{k}\left(C_{\infty}^{*}(x)+\lambda\right) \int_{\Omega} u^{2^{k}} d x
\end{aligned}
$$

Since $u^{2^{k}-2} \leqslant u^{2^{k}}+1, \forall k=1,2, \ldots$ and $\left(d_{1}+\cdots+d_{n}\right)^{r} \leqslant$ $n^{r-1}\left(d_{1}^{r}+\cdots+d_{n}^{r}\right), d_{i} \geqslant 0$ and $r \geqslant 1$ in (18) we obtain

$$
\begin{aligned}
& \frac{d}{d t} \int_{\Omega} u^{2^{k}} d x \leqslant-\frac{\left(2^{k}-1\right)}{2^{k-2}} \vartheta \int_{\Omega} \sum_{i=1}^{n}\left[\frac{\partial\left(u^{2^{k-1}}\right)}{\partial x_{i}}\right]^{2} d x \\
& +2^{k} \tau \int_{\Omega}\left(u^{2^{k}}+1\right) d x+2 B_{\infty}^{*}\left(\int_{\Omega} n \sum_{i=1}^{n}\left[\frac{\partial\left(u^{2^{k-1}}\right)}{\partial x_{i}}\right]^{2} d x\right)^{\frac{1}{2}} \\
& \times\left(\int_{\Omega} u^{2^{k}} d x\right)^{\frac{1}{2}}+2^{k}\left(\zeta+C_{\infty}^{*}(x)+\lambda\right) \int_{\Omega} u^{2^{k}} d x \\
& \leqslant-\frac{\left(2^{k}-1\right)}{2^{k-2}} \vartheta \int_{\Omega} \sum_{i=1}^{n}\left[\frac{\partial\left(u^{2^{k-1}}\right)}{\partial x_{i}}\right]^{2} d x+2^{k}\left(\zeta+\tau+C_{\infty}^{*}(x)\right. \\
& +\lambda) \int_{\Omega} u^{2^{k}} d x+2 B_{\infty}^{*}\left(\int_{\Omega} \sum_{i=1}^{n}\left[\frac{\partial\left(u^{2^{k-1}}\right)}{\partial x_{i}}\right]^{2} d x\right)^{\frac{1}{2}}\left(n \int_{\Omega} u^{2^{k}} d x\right)^{\frac{1}{2}} \\
& +2^{k} \tau|\Omega|
\end{aligned}
$$

Now, using the Cauchy's inequality in (19) with $\bar{\varepsilon}=\frac{\vartheta}{B_{\infty}^{*}}$ we get

$$
\begin{aligned}
& \frac{d}{d t} \int_{\Omega} u^{2^{k}} d x \leqslant-\frac{\left(2^{k}-1\right)}{2^{k-2}} \vartheta \int_{\Omega} \sum_{i=1}^{n}\left[\frac{\partial\left(u^{2^{k-1}}\right)}{\partial x_{i}}\right]^{2} d x \\
& +2^{k}\left(\zeta+\tau+C_{\infty}^{*}(x)+\lambda\right) \int_{\Omega} u^{2^{k}} d x \\
& +2 B_{\infty}^{*}\left(\frac{\bar{\varepsilon}}{2} \int_{\Omega} \sum_{i=1}^{n}\left[\frac{\partial\left(u^{2^{k-1}}\right)}{\partial x_{i}}\right]^{2} d x+\frac{1}{2 \bar{\varepsilon}} n \int_{\Omega} u^{2^{k}} d x\right)+2^{k} \tau|\Omega| \\
& =\left(-\frac{\left(2^{k}-1\right)}{2^{k-2}} \vartheta+\vartheta\right) \int_{\Omega} \sum_{i=1}^{n}\left[\frac{\partial\left(u^{2^{k-1}}\right)}{\partial x_{i}}\right]^{2} d x+\left[2^{k}(\zeta\right. \\
& \left.\left.+\tau+C_{\infty}^{*}(x)+\lambda\right)+\frac{n\left(B_{\infty}^{*}\right)^{2}}{\vartheta}\right] \int_{\Omega} u^{2^{k}}+2^{k} \tau|\Omega| .
\end{aligned}
$$

Since $2 \leqslant \frac{2^{k}-1}{2^{k-2}} \leqslant 4$ for all $k=1,2, \ldots$, we can write $(20)$ as

$$
\begin{aligned}
& \frac{d}{d t} \int_{\Omega} u^{2^{k}} d x \leqslant-\vartheta \int_{\Omega} \sum_{i=1}^{n}\left[\frac{\partial\left(u^{2^{k-1}}\right)}{\partial x_{i}}\right]^{2} d x+\left[2^{k}(\zeta+\tau\right. \\
& \left.\left.+C_{\infty}^{*}(x)+\lambda\right)+\frac{n\left(B_{\infty}^{*}\right)^{2}}{\vartheta}\right] \int_{\Omega} u^{2^{k}}+2^{k} \tau|\Omega|
\end{aligned}
$$

Taking $j=0, p=2, m=1, r=2, q=1, \theta=\frac{n}{n+2}$ in Gagliardo-Nirenberg inequality we obtain

$$
\|v\|_{L^{2}(\Omega)} \leqslant \widetilde{C}\|v\|_{H^{1}(\Omega)}^{\theta}\|v\|_{L^{1}(\Omega)}^{1-\theta}, \forall v \in H^{1}(\Omega),
$$

where $\widetilde{C}=\widetilde{C}(\Omega, n)$. Again, using the Young's inequality in (22) with $m=\frac{1}{\theta}>1$ and $\varepsilon \in(0,1)$ we obtain

$$
\begin{aligned}
\|v\|_{L^{2}(\Omega)}^{2} & \leqslant \frac{1}{m} \varepsilon^{m}\|v\|_{H^{1}(\Omega)}^{2 \theta m}+\frac{m-1}{m} \varepsilon^{\frac{-m}{m-1}} \widetilde{C}^{\frac{2 m}{m-1}}\|v\|_{L^{1}(\Omega)}^{\frac{2 m(1-\theta)}{m-1}} \\
& \leqslant \varepsilon\|v\|_{H^{1}(\Omega)}^{2}+C_{\varepsilon}\|v\|_{L^{1}(\Omega)}^{2},
\end{aligned}
$$

where $C_{\varepsilon}:=\xi \varepsilon^{-\frac{n}{2}-1}$ and $\xi:=\frac{2}{n+2} \widetilde{C}^{n+2}$. From the definition of norm in $H^{1}(\Omega)$ we can see that (23) can be written as

$$
\frac{1-\varepsilon}{\varepsilon}\|v\|_{L^{2}(\Omega)}^{2}-\frac{C_{\varepsilon}}{\varepsilon}\|v\|_{L^{1}(\Omega)}^{2} \leqslant\left\|v_{x}\right\|_{H^{1}(\Omega)}^{2} .
$$

Now, using (24) in (21) with $v=u^{2^{k-1}}$ we get

$$
\begin{aligned}
& \frac{d}{d t} \int_{\Omega} u^{2^{k}} d x \leqslant-\frac{\vartheta(1-\varepsilon)}{\varepsilon} \int_{\Omega} u^{2^{k}} d x+\frac{\vartheta C_{\varepsilon}}{\varepsilon}\left(\int_{\Omega} u^{2^{k}} d x\right)^{2} \\
& \quad+\left[2^{k}\left(\zeta+\tau+C_{\infty}^{*}(x)+\lambda\right)+\frac{n\left(B_{\infty}^{*}\right)^{2}}{\vartheta}\right] \int_{\Omega} u^{2^{k}}+2^{k} \tau|\Omega| \\
& =\left[-\frac{\vartheta(1-\varepsilon)}{\varepsilon}+2^{k}\left(\zeta+\tau+C_{\infty}^{*}(x)+\lambda\right)+\frac{n\left(B_{\infty}^{*}\right)^{2}}{\vartheta}\right] \\
& \quad \times \int_{\Omega} u^{2^{k}} d x+\frac{\vartheta C_{\varepsilon}}{\varepsilon}\left(\int_{\Omega} u^{2^{k}} d x\right)^{2}+2^{k} \tau|\Omega| .
\end{aligned}
$$

Then, fixing $\varepsilon=\varepsilon_{k}, k=1,2, \ldots$ such that

$$
-\frac{\vartheta(1-\varepsilon)}{\varepsilon}+2^{k}\left(\zeta+\tau+C_{\infty}^{*}(x)+\lambda\right)+\frac{n\left(B_{\infty}^{*}\right)^{2}}{\vartheta} \leqslant-2^{k}
$$

and $\varepsilon_{k}=\rho 2^{-k}$ where $\rho$ is a positive constant, we have $\frac{d}{d t} \int_{\Omega} u^{2^{k}} d x \leqslant-2^{k} \int_{\Omega} u^{2^{k}} d x+\mu\left(2^{k}\right)^{\frac{n}{2}+2}\left(\int_{\Omega} u^{2^{k}} d x\right)^{2}+2^{k} \tau|\Omega|$,

where $\mu:=\vartheta \xi \rho^{-\frac{n}{2}-2}$. Applying Lemma 1.2.5 in [5, p. 17] with $y(t)=\int_{\Omega} u^{2^{k}} d x$ in (25) we obtain

$$
\begin{aligned}
& \int_{\Omega} u^{2^{k}} d x \leqslant \max \left\{\int_{\Omega}\left(u^{0}\right)^{2^{k}} d x, \mu\left(2^{k}\right)^{\frac{n}{2}+1}\left(\int_{\Omega} u^{2^{k}} d x\right)^{2}+\tau|\Omega|\right\} \\
& \leqslant \max \left\{\int_{\Omega}\left(u^{0}\right)^{2^{k}} d x, \mu\left(2^{k}\right)^{\frac{n}{2}+1} m_{k-1}^{2^{k}}+\tau|\Omega|\right\},
\end{aligned}
$$


where $m_{k-1}:=\sup _{t \geqslant 0}\left(\int_{\Omega} u^{2^{k-1}} d x\right)^{2^{-k+1}}$. Taking the $2^{k}-$ th root on both sides of (26) and then the supremum on the left hand we get

$$
m_{k} \leqslant \max \left\{\left\|u^{0}\right\|_{L^{2^{k}(\Omega)}},\left(\mu\left(2^{k}\right)^{\frac{n}{2}+1} m_{k-1}^{2^{k}}+\tau|\Omega|\right)^{\frac{1}{2^{k}}}\right\} .
$$

Since, $\quad\left\|u^{0}\right\|_{L^{2^{k}}(\Omega)} \leqslant \sup _{x \in \Omega}\left|u^{0}(x)\right|\left(\int_{\Omega} d x\right)^{\frac{1}{2^{k}}} \leqslant$ $\left\|u^{0}\right\|_{L^{\infty}(\Omega)}|\Omega|^{\frac{1}{2^{k}}}=: \mathscr{K}$, we can see that the first term of (27) is uniform bounded by $\mathscr{K}$, for all $k \in \mathbb{N}$. Now, enlarging $\mu$ to the value

$$
\delta:=\max \left\{\mu, 1, \tau|\Omega|, \mathscr{K}^{2}\right\} .
$$

Enlarging also $m_{1}$ (which is defined by $\sup _{x \in \Omega}\|u(t, \cdot)\|_{L^{2}(\Omega)}$ ) to the value $x_{1}:=\max \left\{m_{1}, 1\right\}$. Then, using (28) in (27) we have

$$
m_{k} \leqslant \max \left\{\mathscr{K},\left(\delta\left(2^{k}\right)^{\frac{n}{2}+1} m_{k-1}^{2^{k}}+\delta\right)^{\frac{1}{2^{k}}}\right\}, k=2,3, \ldots
$$

We can see that the numbers $m_{k}, k=1,2, \ldots$ are bounded by the corresponding $x_{k}$ satisfying the recurrence relation

$$
x_{k}=\max \left\{\mathscr{K},\left(\delta\left(2^{k}\right)^{\frac{n}{2}+1} x_{k-1}^{2^{k}}+\delta\right)^{\frac{1}{2^{k}}}\right\}, k=2,3, \ldots
$$

Since $\delta \geqslant 1$ and $x_{1} \geqslant 1$ we can see that sequence $\left\{x_{k}\right\}_{k \in \mathbb{N}}$ is increasing. Furthermore, for $k=2$ the second term of (29) is bounded because

$$
\begin{aligned}
x_{2} & =\max \left\{\mathscr{K},\left(\delta(2)^{n+2} x_{1}^{4}+\delta\right)^{\frac{1}{4}}\right\} \\
& \leqslant \max \left\{\mathscr{K},\left(2 \delta(2)^{n+2} x_{1}^{4}\right)^{\frac{1}{4}}\right\} \\
& \leqslant \max \left\{\mathscr{K},\left(2 \delta(2)^{n+2}\right)^{\frac{1}{4}} \max \left\{m_{1}, 1\right\}\right\} .
\end{aligned}
$$

By other side, the sequence $\left\{x_{k}\right\}_{k \in \mathbb{N}}$ is dominated by the sequence $\left\{z_{k}\right\}_{k \in \mathbb{N}}$ given by

$$
\left\{\begin{array}{l}
z_{1}=x_{1} \geqslant 1 \\
z_{k}=\left(2 \delta\left(2^{k}\right)^{\frac{n}{2}+1}\right)^{\frac{1}{2^{k}}} z_{k-1}, k=2,3, \ldots
\end{array}\right.
$$

Taking the limit when $k \rightarrow \infty$ we have $x_{\infty}:=\lim _{k \rightarrow \infty} x_{k} \leqslant z_{\infty}:=$ $\lim _{k \rightarrow \infty} z_{k}$. Thus,

$$
\begin{aligned}
& \sup _{t \geqslant 0}\|u(t, \cdot)\|_{L^{\infty}(\Omega)} \leqslant z_{1} \prod_{k=2}^{\infty}\left(2 \delta\left(2^{k}\right)^{\frac{n}{2}+1}\right)^{\frac{1}{2^{k}}} \\
& \leqslant z_{1}\left(\delta(2)^{\frac{n}{2}+2}\right)^{-\frac{1}{2}} \prod_{k=1}^{\infty}(2 \delta)^{\frac{1}{2^{k}}}\left[\prod_{k=1}^{\infty} 2^{\frac{k}{2^{k}}}\right]^{\frac{n}{2}+1} \\
& \leqslant z_{1}\left(\delta(2)^{\frac{n}{2}+2}\right)^{-\frac{1}{2}}(2 \delta)^{\lim _{k \rightarrow \infty}\left(1-\frac{1}{2^{k}}\right)}\left[2^{\lim _{k \rightarrow \infty}}\left(2-\frac{k+2}{2^{k}}\right)\right]^{\frac{n}{2}+1} \\
& \leqslant \delta^{\frac{1}{2}} 2^{\frac{3 n}{4}+1} \max \left\{\sup _{t \geqslant 0}\|u(t, \cdot)\|_{L^{2}(\Omega)}, 1\right\} .
\end{aligned}
$$

Finally we will show that $\sup _{t \geqslant 0}\left\|u\left(t, u^{0}\right)\right\|_{L^{2}(\Omega)} \leqslant \gamma$, where $\gamma>0$. Indeed, similarly as we did above, we multiply the equation of the problem (1) for $u$ and integrate over $\Omega$ we obtain

$$
\begin{gathered}
\int_{\Omega} u_{t} u d x=-\int_{\Omega} \sum_{i, j=1}^{n} a_{i j}(x) \frac{\partial u}{\partial x_{i}} \frac{\partial u}{\partial x_{j}} d x+\int_{\Omega} \sum_{i=1}^{n} b_{i}(x) \frac{\partial u}{\partial x_{i}} u d x \\
+\int_{\Omega}(c(x)+\lambda) u^{2} d x+\int_{\Omega} f(u) u d x
\end{gathered}
$$

From (2) and (12) we get

$$
\begin{aligned}
& \frac{1}{2} \frac{d}{d t} \int_{\Omega} u^{2} d x \leqslant-\vartheta \int_{\Omega} \sum_{i=1}^{n}\left(\frac{\partial u}{\partial x_{i}}\right)^{2} d x+B_{\infty}^{*} \int_{\Omega} \sum_{i=1}^{n} \frac{\partial u}{\partial x_{i}} u d x \\
& \quad+\left(C_{\infty}^{*}+\lambda\right) \int_{\Omega} u^{2} d x+\int_{\Omega}\left(\zeta u^{2}+\tau\right) d x
\end{aligned}
$$

where $B_{\infty}^{*}$ e $C_{\infty}^{*}$ are defined as above. Using Poincare's and Hölder's inequality we obtain

$$
\begin{aligned}
\frac{d}{d t} \int_{\Omega} u^{2} d x & \leqslant-2 \bar{C} \vartheta \int_{\Omega} u^{2} d x+2 B_{\infty}^{*}\left(\int_{\Omega} \sum_{i=1}^{n}\left(\frac{\partial u}{\partial x_{i}}\right)^{2} d x\right)^{\frac{1}{2}} \\
& \times\left(n \int_{\Omega} u^{2} d x\right)^{\frac{1}{2}}+2\left(C_{\infty}^{*}+\lambda\right) \int_{\Omega} u^{2} d x \\
& +2 \zeta \int_{\Omega} u^{2} d x+2 \tau|\Omega|
\end{aligned}
$$

By Cauchy's inequality with $\varepsilon_{0}>0$ in (31), we have

$$
\begin{aligned}
\frac{d}{d t} \int_{\Omega} u^{2} d x & \leqslant 2\left(-\bar{C} \vartheta+\frac{\bar{C} B_{\infty}^{*} \varepsilon_{0}}{2}+C_{\infty}^{*}+\lambda+\zeta+\frac{n B_{0}^{*}}{\varepsilon_{0}}\right) \\
& \times \int_{\Omega} u^{2} d x+2 \tau|\Omega|
\end{aligned}
$$

where in the last inequality we have used Poincaré's inequality.

Choosing $\varepsilon_{0}$ such that

$$
-\bar{C} \vartheta+\frac{\bar{C} B_{\infty}^{*} \varepsilon_{0}}{2}+C_{\infty}^{*}+\lambda+\zeta+\frac{n B_{\infty}^{*}}{\varepsilon_{0}} \leqslant-1 .
$$

Thus, in (32) we have

$$
\frac{d}{d t} \int_{\Omega} u^{2} d x \leqslant-2 \int_{\Omega} u^{2} d x+2 \tau|\Omega| .
$$

Using Lemma 1.2.4 in [5, p. 17] to (33), we get

$$
\sup _{t \geqslant 0}\left\|u\left(t, u^{0}\right)\right\|_{L^{2}(\Omega)} \leqslant \max \left\{\left\|u^{0}\right\|_{L^{2}(\Omega)}, \tau|\Omega|\right\} .
$$

Therefore, using (34) in (30) we obtain

$$
\sup _{t \geqslant 0}\left\|u\left(t, u^{0}\right)\right\|_{L^{\infty}(\Omega)} \leqslant \delta^{\frac{3}{2}} 2^{\frac{3 n}{4}+1} \max \left\{\left\|u^{0}\right\|_{L^{2}(\Omega)}, 1\right\} .
$$

Thus we get the result. 
Lemma 6.Let $f: \mathbb{R} \rightarrow \mathbb{R}$ be a function of class $C^{2}(\mathbb{R})$ satisfying (D). Then given $\varepsilon>0$ there exists $m_{\varepsilon}=: m>0$ such that

$$
v f(v) \leqslant \varepsilon v^{2}+m
$$

for all $v \in \mathbb{R}$.

Proof. From the assumption (D), follows that is there is $M>0$ such that $v f(v) \leqslant \varepsilon v^{2}$, for all $|v| \geqslant M$. Since the set $\{x \in \bar{\Omega}:|v| \leqslant M\}$ is bounded in $\mathbb{R}^{n}$ where we take $v=v(x)$ and $f$ be a continuous function. Thus, we have $|v f(v)| \leqslant m_{\varepsilon}$. Therefore, joining these two facts follow the result. $\square$

\section{Local and global solution}

Lemma 7(Local solution). Under the growth assumption (C) the problem (3) has local solution in $X^{1 / 2}$.

Proof. By Lemma 3 and Lemma 2 we can see that the hypothesis of Theorem 2.1.1 in [5, p. 62] are satisfied, thus we have the result.

Theorem 1(Bounded global solution). Assume the growth conditions (C) and dissipation (D) holds, then the solution of the problem (3) with $u^{0} \in B_{R}=\left\{v \in X^{1 / 2}:\|v\|_{H_{0}^{1}(\Omega)} \leq R\right\}$ is define globally and there exist a constant $K_{1}>0$ such that

$$
\limsup _{t \rightarrow \infty}\left\|u\left(t, u^{0}\right)\right\|_{H_{0}^{1}(\Omega)} \leq K_{1} .
$$

Proof. From (13) and Lemma 5, we obtain that $u \in L^{\infty}(\Omega)$, then $\|F(u)\|_{X} \leq C\left(\|u\|_{L^{\infty}(\Omega)}\right)$. Thus, if $u^{0} \in X^{\alpha}$, then $u$ is a local solution in $X^{\alpha}$ of the problem (3) satisfying the constants variation formula. Since, the operator $A$ is sectorial positive, we have

$$
\begin{aligned}
& \left\|u\left(t, u^{0}\right)\right\|_{X^{\alpha}} \leq\left\|A^{\alpha} e^{-A t} u^{0}\right\|_{X} \\
& \quad+\int_{0}^{t}\left\|A^{\alpha} e^{-A(t-s)}\right\|_{\mathscr{L}(X)}\left\|F\left(u\left(s, u^{0}\right)\right)\right\|_{X} d s \\
& \leq c_{0} e^{-\beta t}\left\|u^{0}\right\|_{X^{\alpha}}+\int_{0}^{t} c_{1}(t-s)^{-\alpha} e^{-\beta(t-s)}\left\|F\left(u\left(s, u^{0}\right)\right)\right\|_{X} d s \\
& \leq c_{0}\left\|u^{0}\right\|_{X^{\alpha}}+c_{1} C\left(\|u\|_{L^{\infty}(\Omega)}\right) \int_{0}^{t}(t-s)^{-\alpha} d s,
\end{aligned}
$$

where $c_{0}, c_{1}$ are positive constants with $\alpha \in(0,1)$ and $\operatorname{Re} \sigma(A)>\beta>0$. It follows that $u\left(t, u^{0}\right)$ in the norm $X^{\alpha}$ is limited to finite intervals of time with $\alpha<1$. Therefore, for $\alpha=1 / 2$ a solution is global.

As before, using the formula of the constants variation and since $\left\|u\left(t, u^{0}\right)\right\|_{L^{\infty}(\Omega)} \leq K_{\infty}$ for all $t \geq 0$, we obtain

$$
\begin{gathered}
\left\|u\left(t, u^{0}\right)\right\|_{X^{\alpha}} \leq c_{\alpha} t^{-\alpha} e^{-\beta t}\left\|u^{0}\right\|_{X} \\
\quad+\int_{0}^{t} c_{1}(t-s)^{-\alpha} e^{-\beta(t-s)} C\left(K_{\infty}\right) d s \\
\leq c_{\alpha} t^{-\alpha} e^{-\beta t}\left\|u^{0}\right\|_{L^{\infty}(\Omega)}+c_{1} C\left(K_{\infty}\right) \int_{0}^{t}(t-s)^{-\alpha} e^{-\beta(t-s)} d s .
\end{gathered}
$$

From this, it follows

$$
\begin{aligned}
\limsup _{t \rightarrow \infty}\left\|u\left(t, u^{0}\right)\right\|_{X^{\alpha}} & \leq \limsup _{t \rightarrow \infty} C\left(K_{\infty}\right) \int_{0}^{t} r^{-\alpha} e^{-\beta r} d s \\
& =c_{1} C\left(K_{\infty}\right) \Gamma(1-\alpha) \beta^{\alpha-1}=: K_{1} . \square
\end{aligned}
$$

Let $\{T(t, \cdot): t \geqslant 0\}$ be a semigroup in $X^{1 / 2}$ given by

$$
T\left(t, u^{0}\right)=u\left(t, u^{0}\right), \forall t \geqslant 0
$$

where $u$ is the unique global solution of (3). This semigroup $\{T(t, \cdot): t \geqslant 0\}$ is a $C_{0}$-semigroup in $X^{1 / 2}$.

As a consequence of Theorem 1 results

Corollary 1.Under the hypotheses of Theorem 1, it follows that semigroup $\left\{T\left(t, u^{0}\right): t \geq 0\right\}$ is point dissipative.

\section{Existence of global attractor}

Theorem 2.The $C_{0}$-semigroup $\{T(t, \cdot): t \geqslant 0\}$ associated to the problem (3) has global attractor $\mathscr{A}$ in $X^{1 / 2}$.

Proof. We show first that $C_{0}$-semigroup $\left\{T\left(t, u^{0}\right): t \geq 0\right\}$ is compact in $X^{1 / 2}$. In fact, we see that the resolvent of $A$ is compact, since $X^{1}=D(A)$ is embedding compactly in $X=L^{2}(\Omega)$ and of the Proposition 4.25 in [6, p. 118], the result follows. Now, using the Theorem 3.3.1 in [5, p. 80], we have the $C_{0}$-semigroup $\left\{T\left(t, u^{0}\right): t \geq 0\right\}$ is compact in $X^{1 / 2}$. Finally, by Corollary's 1 and 1.1 .6 in [5, p.13] the result follows.

As a consequence of Lemma 5 and Lemma 6, we have

Theorem 3.Assuming the same hipotheses of Lemma 13, we have

$$
\sup _{u \in \mathscr{A}}\|u\|_{L^{\infty}(\Omega)}<K_{0}
$$

where $K_{0}=K_{0}\left(\Omega, n, \zeta, \tau,\left\|u^{0}\right\|_{L^{\infty}(\Omega)}\right)$ is a positive constant.

\section{Acknowledgement}

The first author was partially supported by FAPESP(Brazil) through the research grant 2009/08088-9 and the second author was partially supported by FAPESP(Brazil) through the research grant 2009/08435-0. 


\section{References}

[1] Alikakos, N. D. An application of the invariance principle to reaction-diffusion equations. J. Differential Equations, 33, 201-225 (1979).

[2] Arrieta, J. M.; Carvalho, A. N.; Rodríguez-Bernal, A. Parabolic problems with nonlinear boundary conditions and critical nonlinearities. J. Differential Equations, 156, 376406 (1999).

[3] Arrieta, J. M.; Carvalho, A. N.; Rodríguez-Bernal, A. Attractors of parabolic problems with nonlinear boundary conditions. Uniform bounds. Comm. Partial Differential Equations, 25, 1-37 (2000).

[4] Brezis, H. Functional analysis, Sobolev spaces and partial differential equations. Universitext. Springer, New York, (2011).

[5] Cholewa, J. W.; Dlotko, T. Global attractors in abstract parabolic problems. London Mathematical Society Lecture Note Series, Cambridge University Press, Cambridge, 278, (2000).

[6] Engel, K. J.; Nagel, R. One-parameter semigroups for linear evolution equations, Graduate Texts in Mathematics, Springer-Verlag, New York, 194, (2000).

[7] Friedman, A. Partial Differential Equations, Dover Publications, Inc. Mineola, New York, (2008).

[8] Hale, J. K. Asymptotic behavior of dissipative systems. Math. Surveys Monogr., Amer. Math. Soc., 25, (1988).

[9] Henry, D. Geometric theory of semilinear parabolic equations, Lect. Notes in Math., Springer, 840, (1981).

[10] Raugel, G. Global attractors in partial differential equations. Handbook of dynamical systems, North-Holland, Amsterdam, 2, 885-982 (2002).

[11] Rudin, W. Functional analysis. Second edition. International Series in Pure and Applied Mathematics. McGraw-Hill, Inc., New York, (1991).

[12] Zheng, S. Nonlinear evolution equations. Chapman \& Hall/CRC Monographs and Surveys in Pure and Applied Mathematics, Chapman \& Hall/CRC, Boca Raton, FL, 133, (2004).

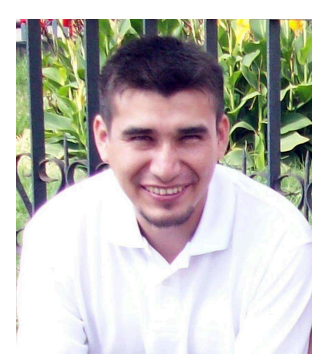

Rodiak Nicolai Figueroa López received the $\mathrm{PhD}$ degree in Mathematics (2013) from Universidade Estadual Paulista (UNESP) at São José do Rio Preto. His research interests area in the study of the asymptotic dynamics of parabolic partial differential equations and their applications, domain's discretization via the finite element method, the convergence compact operators and semigroups theory.

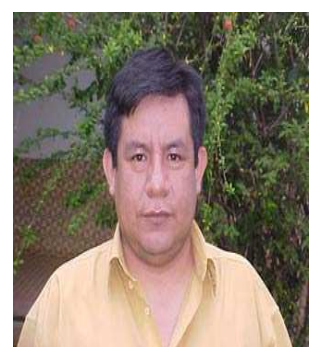

Germán Jesús Lozada Cruz, $\mathrm{PhD}$ in Mathematics (2001) from University of São Paulo at São Carlos. Assistant professor at the Departament of Mathematics of State University of São Paulo. Has experience in Mathematics with emphasis in Partial Differential Equations. The areas of interest are asymptotic behavior of reaction diffusion equations in dumbbell domains, existence of attractors and continuity of attractors with relation to small parameter.. 